\title{
Squaring down LTI systems: A geometric approach ${ }^{\text {ts }}$
}

\author{
Lorenzo Ntogramatzidis $^{\mathrm{a}, *}$, Domenico Prattichizzo ${ }^{\mathrm{b}}$ \\ ${ }^{a}$ Department of Electrical and Electronic Engineering, The University of Melbourne, Parkville VIC 3010, Australia \\ ${ }^{\mathrm{b}}$ Dipartimento di Ingegneria dell'Informazione, Università di Siena, via Roma 56, I-53100 Siena, Italy
}

Received 4 October 2005; received in revised form 14 September 2006; accepted 5 October 2006

Available online 28 November 2006

\begin{abstract}
In this paper, the problem of reducing a given LTI system into a left or right invertible one is addressed and solved with the standard tools of the geometric control theory. First, it will be shown how an LTI system can be turned into a left invertible system, thus preserving key system properties like stabilizability, phase minimality, right invertibility, relative degree and infinite zero structure. Moreover, the additional invariant zeros introduced in the left invertible system thus obtained can be arbitrarily assigned in the complex plane. By duality, the scheme of a right inverter will be derived straightforwardly. Moreover, the squaring down problem will be addressed. In fact, when the left and right reduction procedures are applied together, a system with an unequal number of inputs and outputs is turned into a square and invertible system. Furthermore, as an example it will be shown how these techniques may be employed to weaken the standard assumption of left invertibility of the plant in many optimization problems.

(C) 2006 Elsevier B.V. All rights reserved.
\end{abstract}

Keywords: Output-nulling and input-containing subspaces; Left and right invertibility; Invariant zeros; Squaring down; Linear quadratic regulator

\section{Introduction}

In the last two decades, many contributions on the conversion of LTI systems into left and right invertible systems have been presented in the literature, motivated by the fact that several design and synthesis procedures require that the plant is left or right invertible or, in some cases, square and invertible, $[12,14,21]$. It is an easily established fact that the introduction of a suitable static compensator at the input of a nonleft invertible system yields a left invertible system, for example by reducing the number of inputs of the given system. This is the simplest structure of a left inverter, and was presented in the first papers that appeared in the literature on this issue, see [13] and references therein. The main drawback of that approach, however, was then shown to be the inevitable introduction of nonassignable additional invariant zeros in the resulting

\footnotetext{
Partially supported by the ministry of higher education of Italy (MIUR), under the project Identification and Control of Industrial Systems, by The University of Melbourne (MRGS) and the Australian Research Council.

* Corresponding author. Tel.: +6138344 7436; fax: +61383446678.

E-mail addresses: lnt@ee.unimelb.edu.au (L. Ntogramatzidis), prattichizzo@dii.unisi.it (D. Prattichizzo).
}

system, [8]. Hence, in the case when any of these new invariant zeros was nonminimum phase, the performances of the closedloop behaviour were heavily affected.

It was then observed that in order to preserve phase minimality after the left (right) reduction, the use of dynamic pre (post)-compensators was mandatory, $[2,18,16,12,20]$. In particular, in $[18,16]$, the design procedure of left and right inverters was carried out for strictly and nonstrictly proper systems, respectively, by the exploitation of the so-called special coordinate basis for the representation of the plant (see also [17] for details). In this way, it was proven that these compensators enabled the introduction of additional nonminimum phase zeros to be avoided. Moreover, some important features of the given system, such as stabilizability and infinite zero structure, were preserved in the resulting system.

The procedure proposed in this paper provides a new solution to the left and right reduction of a continuous or discrete-time LTI system. In particular, the first is based on the introduction of a static compensator at the input of the given system, working jointly with an algebraic state-feedback unit, whose purpose is that of arbitrarily assigning all the additional invariant zeros introduced in the left invertible system thus obtained. This compensation scheme preserves the simplicity of 
the aforementioned static compensators, but avoids the introduction of possibly nonminimum phase zeros. Moreover, the proposed left inverter ensures that the additional invariant zeros are all assignable in the complex plane, while the stabilizability and the functional controllability of the original system are preserved; in particular, if the given system is right invertible, so is the resulting system, and the relative degree does not change. Furthermore, the infinite zero structure of the original system is also preserved.

A straightforward dualization of the aforementioned procedure solves the problem of reducing a system into a right invertible one, thus preserving key properties of the original system, such as phase minimality, detectability, functional observability and infinite zero structure.

Differently from the other approaches presented in the literature, the geometric setting herein employed is coordinate-free in nature, and leads to intuitive results without the need of resorting to changes of basis like the SCB, which on the one hand clearly displays the inner structure of the underlying system, but, on the other hand, is not computationally robust as recently shown in [5]. A further contribution of this work is that of generalizing the concepts of input unobservability subspace and output reachability subspace introduced in [3] to nonpurely dynamical systems.

An important field of application of the material herein developed concerns the linear quadratic optimization. As is well-known, in many LQ, $H_{2}$ and $H_{\infty}$ problems the standard assumption of left invertibility of the system is introduced, in order to ensure the uniqueness of the optimal solution, $[9,14,21]$. Moreover, in the discrete-time case this assumption is necessary for the discrete-time algebraic Riccati equation DARE to admit a stabilizing solution. In Section 6 it will be shown how the left inversion technique herein described can be employed to derive a parametrization of all the solutions of the infinite-horizon LQ regulator when the underlying system is not left invertible. This technique therefore constitutes a useful tool for the solution of discrete-time singular LQ problems, in the case where the associated extended symplectic pencil is not regular, thus avoiding the need of resorting to the solution of the so-called generalized algebraic Riccati equation. Singular LQ problems are of importance in several fields. They were first investigated in relation to the occurrence of singular arcs in the aerospace field in the early 1960s. For more detailed information on the history of this problem see [11,6] and the references therein. Moreover, the need to solve singular problems naturally arise in the solution of $\mathrm{H}_{2}$ rejection problems of strictly proper systems, [19].

Notation: Throughout this paper, we denote by $\mathbb{N}$ the positive integers including zero. The symbol $0_{n}$ denotes the origin of the vector space $\mathbb{R}^{n}$. The image and the kernel of matrix $A \in \mathbb{R}^{n \times m}$ are denoted by $\operatorname{im} A$ and $\operatorname{ker} A$, respectively. Given a subspace $\mathscr{Y}$ of $\mathbb{R}^{n}$, the symbol $A^{-1} \mathscr{Y}$ stands for the inverse image of $\mathscr{Y}$ with respect to the linear map $A$, while $\mathscr{Y}^{\perp}$ represents the orthogonal complement of $\mathscr{Y}$. Denote by $A^{\top}$ the transpose of $A$. The symbol $\sigma(A)$ denotes the spectrum of $A$.

The restriction of a linear map $A$ to the $A$-invariant subspace $\mathscr{J}$ is written $\left.A\right|_{\mathscr{J}}$; the eigenvalues of $A$ restricted to $\mathscr{J}$ are denoted by $\sigma(A \mid \mathscr{J})$. If $\mathscr{J}_{1}$ and $\mathscr{J}_{2}$ are $A$-invariant subspaces and $\mathscr{J}_{1} \subseteq \mathscr{J}_{2}$, the mapping induced by $A$ on the quotient space $\mathscr{J}_{2} / \mathscr{J}_{1}$ is denoted by $\left(A \mid \mathscr{J}_{2} / \mathscr{J}_{1}\right)$.

In what follows, whether the underlying system evolves in continuous or discrete time is irrelevant and, accordingly, the time index set of any signal is denoted by $\mathbb{T}$, on the understanding that this represents either $\mathbb{R}^{+}$in the continuous time or $\mathbb{N}$ in the discrete time. The symbol $\mathbb{C}_{g}$ denotes either the open left-half complex plane $\mathbb{C}^{-}$in the continuous time or the open unit disc $\mathbb{C}^{\circ}$ in the discrete time.

\section{Problem statement}

Consider an LTI system $\Sigma$ described by

$$
\begin{aligned}
& \rho x(t)=A x(t)+B u(t), \\
& y(t)=C x(t)+D u(t),
\end{aligned}
$$

where, for all $t \in \mathbb{T}, x(t) \in \mathbb{R}^{n}$ is the state, $u(t) \in \mathbb{R}^{m}$ is the control input, $y(t) \in \mathbb{R}^{p}$ is the output, $A, B, C$ and $D$ are real constant matrices of suitable sizes, i.e., $A \in \mathbb{R}^{n \times n}, B \in \mathbb{R}^{n \times m}$, $C \in \mathbb{R}^{p \times n}$ and $D \in \mathbb{R}^{p \times m}$. The operator $\rho$ denotes either the time derivative in the continuous time, i.e., $\rho x(t)=\dot{x}(t)$, or the unit time shift in the discrete time, i.e., $\rho x(t)=x(t+1)$.

With no loss of generality it is assumed that $\left[\begin{array}{ll}B^{\top} & D^{\top}\end{array}\right]^{\top}$ has linearly independent columns and $\left[\begin{array}{ll}C & D\end{array}\right]$ has linearly independent rows. Indeed, if for example $\left[B^{\top} D^{\top}\right]^{\top}$ has nontrivial kernel, there exists a subspace $\mathscr{U}_{0}$ of the input space that does not influence the state dynamics and the output function. Then, by performing a suitable (orthogonal) change of basis in the input space, we may eliminate $\mathscr{U}_{0}$ and obtain an equivalent problem for which this condition is satisfied.

From now on we will concisely identify $\Sigma$ with the quadruple $(A, B, C, D)$.

The aim of this paper is that of presenting a design procedure based on strict geometric techniques for the conversion of the given system $\Sigma$ to a left (resp., right) invertible system, i.e., a transformation involving $\Sigma$ whose purpose is that of obtaining a new system which is left (resp., right) invertible, and preserving some important properties characterizing the original system $\Sigma$, such as phase minimality, stabilizability (resp., detectability), functional controllability (resp., functional observability) and infinite zero structure.

When the geometric left and right reduction procedures are applied together over an arbitrary (possibly degenerate) system, we obtain a new system $\Sigma_{\text {sq }}$ having the same number of inputs and outputs and such that its transfer function matrix is invertible over the set of rational matrices.

\section{Geometric preliminaries}

For the readers' convenience, in this section some fundamental definitions and results of the geometric approach which will be used in the sequel are recalled (for a detailed discussion on the topics herein introduced we refer to [4,22,23]). First, we define an output-nulling subspace $\mathscr{V}_{\Sigma}$ of $\Sigma$ as a subspace 
of $\mathbb{R}^{n}$ satisfying

$\left[\begin{array}{l}A \\ C\end{array}\right] \mathscr{V}_{\Sigma} \subseteq\left(\mathscr{V}_{\Sigma} \times 0_{p}\right)+\operatorname{im}\left[\begin{array}{l}B \\ D\end{array}\right]$

The set of output-nulling subspaces of $\Sigma$ is an upper semilattice with respect to subspace addition. In other words, for any given pair of output-nulling subspaces $\mathscr{V}_{\Sigma}^{\prime}$ and $\mathscr{V}_{\Sigma}^{\prime \prime}$, their sum $\mathscr{V}_{\Sigma}^{\prime}+\mathscr{V}_{\Sigma}^{\prime \prime}$ is the smallest output-nulling subspace of $\Sigma$ containing both $\mathscr{V}_{\Sigma}^{\prime}$ and $\mathscr{V}_{\Sigma}^{\prime \prime}$. Thus, the sum of all the output-nulling subspaces of $\Sigma$ is the largest output-nulling subspace of $\Sigma$, and will be herein denoted by $\mathscr{V}_{\Sigma}^{*}$. The subspace $\mathscr{V}_{\Sigma}^{*}$ represents the set of all initial states of (1) for which an input function exists such that the corresponding state-trajectory lies entirely on $\mathscr{V}_{\Sigma}^{*}$ and the corresponding output function is identically zero. In the case when the direct feedthrough matrix $D$ is zero, $\mathscr{V}_{\Sigma}^{*}$ reduces to the maximal $(A, B)$-controlled invariant subspace contained in the null-space of matrix $C,[4,23]$. In the following lemma, the most important properties of the output-nulling subspaces are presented.

\section{Lemma 1. The following results hold:}

(i) The subspace $\mathscr{V}_{\Sigma}$ is output nulling for $\sum$ if and only if a matrix $F \in \mathbb{R}^{m \times n}$ exists such that

$$
\begin{aligned}
& (A+B F) \mathscr{V}_{\Sigma} \subseteq \mathscr{V}_{\Sigma}, \\
& \mathscr{V}_{\Sigma} \subseteq \operatorname{ker}(C+D F) .
\end{aligned}
$$

(ii) The subspace $\mathscr{V}_{\Sigma}^{*}$ is the largest subspace for which a matrix $F \in \mathbb{R}^{m \times n}$ exists such that (3) holds:

(iii) The sequence of subspaces $\left(\mathscr{V}_{\Sigma}^{i}\right)_{i \in \mathbb{N}}$ described by the recurrence

$\mathscr{V}_{\Sigma}^{0}=\mathbb{R}^{n}$

$\mathscr{V}_{\Sigma}^{i}=\left[\begin{array}{l}A \\ C\end{array}\right]^{-1}\left(\left(\mathscr{V}_{\Sigma}^{i-1} \times 0_{p}\right)+\operatorname{im}\left[\begin{array}{c}B \\ D\end{array}\right]\right), \quad i=\mathbb{N} \backslash 0$,

is monotonically nonincreasing. Moreover, there exists $k \leqslant n-1$ such that $\mathscr{V}_{\Sigma}^{k+1}=\mathscr{V}_{\Sigma}^{k}$. For such $k$ there holds $\mathscr{V}_{\Sigma}^{*}=\mathscr{V}_{\Sigma}^{k}$.

Any matrix $F$ satisfying (3) will be referred to as a friend of the output-nulling subspace $\mathscr{V}_{\Sigma}$. The dual concept is the input-containing subspace: a subspace $\mathscr{S}_{\Sigma}$ is said to be input containing if it satisfies

$\left[\begin{array}{ll}A & B\end{array}\right]\left(\left(\mathscr{S}_{\Sigma} \times \mathbb{R}^{m}\right) \cap \operatorname{ker}\left[\begin{array}{ll}C & D\end{array}\right]\right) \subseteq \mathscr{S}_{\Sigma}$

The set of all input-containing subspaces of $\Sigma$ is a lower semilattice with respect to the subspace intersection. Hence, for any given pair of input-containing subspaces $\mathscr{S}_{\Sigma}^{\prime}$ and $\mathscr{S}_{\Sigma}^{\prime \prime}$, their intersection $\mathscr{S}_{\Sigma}^{\prime} \cap \mathscr{S}_{\Sigma}^{\prime \prime}$ is the largest input-containing subspace of $\Sigma$ contained in both $\mathscr{S}_{\Sigma}^{\prime}$ and $\mathscr{S}_{\Sigma}^{\prime \prime}$. Thus, the intersection of all input-containing subspaces of $\Sigma$ is the smallest inputcontaining subspace of $\Sigma$, and will be denoted by $\mathscr{S}_{\Sigma}^{*}$. The counterpart of Lemma 1 for input-containing subspaces is as follows.
Lemma 2. The following results hold:

(i) The subspace $\mathscr{S}_{\Sigma}$ is input containing for $\Sigma$ if and only if a matrix $G \in \mathbb{R}^{n \times p}$ exists such that

$$
(A+G C) \mathscr{S}_{\Sigma} \subseteq \mathscr{S}_{\Sigma},
$$$$
\mathscr{S}_{\Sigma} \supseteq \operatorname{im}(B+G D) \text {. }
$$

(ii) The subspace $\mathscr{S}_{\Sigma}^{*}$ is the smallest subspace for which a matrix $G \in \mathbb{R}^{n \times p}$ exists such that (6) holds:

(iii) The sequence of subspaces $\left(\mathscr{S}_{\Sigma}^{i}\right)_{i \in \mathbb{N}}$ described by the recurrence

$\mathscr{S}_{\Sigma}^{0}=0_{n}$,

$\mathscr{S}_{\Sigma}^{i}=\left[\begin{array}{ll}A & B\end{array}\right]\left(\left(\mathscr{S}_{\Sigma}^{i-1} \times \mathbb{R}^{m}\right) \cap \operatorname{ker}\left[\begin{array}{ll}C & D\end{array}\right]\right), \quad i \in \mathbb{N} \backslash 0$,

is monotonically nondecreasing. Moreover, there exists $k \leqslant n-1$ such that $\mathscr{S}_{\Sigma}^{k+1}=\mathscr{S}_{\Sigma}^{k}$. For such $k$ there holds $\mathscr{S}_{\Sigma}^{*}=\mathscr{S}_{\Sigma}^{k}$.

A matrix $G$ satisfying (6) will be referred to as a friend of the input-containing subspace $\mathscr{S}_{\Sigma}$. The third fundamental subspace that we need to define is the output-nulling reachability subspace on the output-nulling subspace $\mathscr{V}_{\Sigma}$, denoted by $\mathscr{R}_{\Sigma}$ : let $F \in \mathbb{R}^{m \times n}$ be a friend of the output-nulling subspace $\mathscr{V}_{\Sigma}$. The output-nulling reachability subspace $\mathscr{R}_{\Sigma}$ on $\mathscr{V}_{\Sigma}$ is the smallest $(A+B F)$-invariant subspace of $\mathbb{R}^{n}$ containing the subspace $\mathscr{V}_{\Sigma} \cap B$ ker $D$. We denote by $\mathscr{R}_{\Sigma}^{*}$ the output-nulling reachability subspace on $\mathscr{V}_{\Sigma}^{*}$. The following relation holds:

$\mathscr{R}_{\Sigma}^{*}=\mathscr{V}_{\Sigma}^{*} \cap \mathscr{S}_{\Sigma}^{*}$.

This identity was first proved for strictly proper systems in [15]. A proof of this fact for systems with direct feedthrough can be found in [22, Theorem 8.22]. The subspace $\mathscr{R}_{\Sigma}^{*}$ can be thought as the locus of all the initial states that are reachable in finite time from the origin while mantaining the corresponding output equal to zero.

It is a well-known fact that, for any friend $F$ of $\mathscr{V}_{\Sigma}^{*}, F$ is also a friend of $\mathscr{R}_{\Sigma}^{*}$. For any friend $F$ of $\mathscr{V}_{\Sigma}^{*}$, the eigenvalues of $(A+$ $B F)$ restricted to $\mathscr{V}_{\Sigma}^{*}$, i.e. $\sigma\left(A+B F \mid \mathscr{V}_{\Sigma}^{*}\right)$, are split into two sets: the eigenvalues of $\left(A+B F \mid \mathscr{R}_{\Sigma}^{*}\right)$ are all freely assignable by a suitable choice of the friend $F$ of $\mathscr{V}_{\Sigma}^{*}$. The eigenvalues of $\left(A+B F \mid \mathscr{V}_{\Sigma}^{*} / \mathscr{R}_{\Sigma}^{*}\right)$ are fixed, i.e., they do not depend on the choice of the friend $F$ of $\mathscr{V}_{\Sigma}^{*}$ : they are the invariant zeros of $\Sigma$, [1]. In symbols

$\mathscr{Z}(\Sigma)=\sigma\left(A+B F \mid \frac{\mathscr{V}_{\Sigma}^{*}}{\mathscr{R}_{\Sigma}^{*}}\right)$.

Now, consider the subspace $\mathscr{Q}_{\Sigma}^{*}:=\mathscr{V}_{\Sigma}^{*}+\mathscr{S}_{\Sigma}^{*}$, which is dual to $\mathscr{R}_{\Sigma}^{*}$. For any friend $G$ of $\mathscr{S}_{\Sigma}^{*}, G$ is also a friend of $\mathscr{Q}_{\Sigma}^{*}$. Moreover, the eigenvalues of $\left(A+G C \mid \mathbb{R}^{n} / \mathscr{2}_{\Sigma}^{*}\right)$ are all freely assignable by a suitable choice of $G$, while the eigenvalues of $\left(A+G C \mid \mathscr{Q}_{\Sigma}^{*} / \mathscr{S}_{\Sigma}^{*}\right)$ are fixed, and are the invariant zeros of $\Sigma$. Hence, the identity $\mathscr{Z}(\Sigma)=\sigma\left(A+B F \mid \mathscr{V}_{\Sigma}^{*} / \mathscr{R}_{\Sigma}^{*}\right)=\sigma(A+$ $\left.G C \mid \mathscr{Q}_{\Sigma}^{*} / \mathscr{S}_{\Sigma}^{*}\right)$ holds. Now we briefly recall the geometric definition and the basic properties of left and right invertible systems. 
Definition 1. The system $\Sigma$ is said to be left invertible if $\mathscr{R}_{\Sigma}^{*}=$ $\mathscr{V}_{\Sigma}^{*} \cap \mathscr{S}_{\Sigma}^{*}=0_{n}$.

Stated differently, we may say that $\Sigma$ is left invertible if no pair of distinct input functions give rise to the same output function with zero initial condition. For this reason, the left invertibility of a system $\Sigma$ is sometimes alternatively referred to as functional observability. The following theorem provides necessary and sufficient conditions for left invertibility.

Lemma 3. System $\Sigma$ is left invertible if and only if any of the following statements hold true:

1. the transfer function matrix $G_{\Sigma}(s)$ associated with $\Sigma$ is a left invertible rational matrix;

2. $\mathscr{V}_{\Sigma}^{*} \cap B$ ker $D=0_{n}$;

3. $B^{-1} \mathscr{V}_{\Sigma}^{*} \cap \operatorname{ker} D=0_{m}$.

By extending straightforwardly a result presented in $[3$, Theorem 3] to nonstrictly proper systems, we can say that if $\Sigma$ is not left invertible, the input function corresponding to a given response (obtained with zero initial condition) can only be determined modulo $B^{-1} \mathscr{V}_{\Sigma}^{*} \cap \operatorname{ker} D$. The subspace $B^{-1} \mathscr{V}_{\Sigma}^{*} \cap \operatorname{ker} D$ is therefore called input unobservability subspace.

Definition 2. The system $\Sigma$ is said to be right invertible if $\mathscr{2}_{\Sigma}^{*}=\mathscr{V}_{\Sigma}^{*}+\mathscr{S}_{\Sigma}^{*}=\mathbb{R}^{n}$.

Stated differently, we may say that $\Sigma$ is right invertible if for any arbitrarily assigned impulsive-smooth output, there exists an impulsive-smooth input yielding that output with zero initial condition. For this reason, the right invertibility of a system $\Sigma$ is sometimes alternatively referred to as functional controllability of $\Sigma$.

Lemma 4. System $\Sigma$ is right invertible if and only if any of the following statements hold true:

1. the transfer function matrix $G_{\Sigma}(s)$ associated with $\Sigma$ is a right invertible rational matrix;

2. $\mathscr{S}_{\Sigma}^{*}+C^{-1} \operatorname{im} D=\mathbb{R}^{n}$;

3. $C \mathscr{S}_{\Sigma}^{*}+\operatorname{im} D=\mathbb{R}^{p}$.

If $\Sigma$ is not right invertible, the output function can be imposed modulo any complement of the subspace $C \mathscr{S}_{\Sigma}^{*}+\operatorname{im} D$, [3, Theorem 4]. Thus, the subspace $C \mathscr{S}_{\Sigma}^{*}+\operatorname{im} D$ is called output reachability subspace. Now, define $v_{i}:=\operatorname{dim}\left(\mathscr{V}_{\Sigma}^{*}+\right.$ $\left.\mathscr{S}_{\Sigma}^{i}\right)-\operatorname{dim}\left(\mathscr{V}_{\Sigma}^{*}+\mathscr{S}_{\Sigma}^{i-1}\right)$, for $i \in \mathbb{N}$, and let $\rho_{i}:=\operatorname{card}\{j \in$ $\left.\mathbb{N} \mid v_{j} \geqslant i\right\}$. The $\rho_{i}$ are the orders of the zeros at infinity of $\Sigma$. The supremal value of the orders of the zeros at infinity is called relative degree of the system $\Sigma$. It is an easily established fact that in the particular case when $\Sigma$ is right invertible, the relative degree of $\Sigma$ corresponds to the least integer $k \in \mathbb{N}$ such that $\mathscr{V}_{\Sigma}^{*}+\mathscr{S}_{\Sigma}^{k+1}=\mathbb{R}^{n}$. Dually, if $\Sigma$ is left invertible, the relative degree of $\Sigma$ equals the least integer $k \in \mathbb{N}$ for which $\mathscr{S}_{\Sigma}^{*} \cap \mathscr{V}_{\Sigma}^{k+1}=0_{n}$. The original geometric definition for the orders ofthe zeros at infinity for strictly proper systems comes back to [7]; its extension to nonstrictly proper systems has been presented in [1, Theorem 4].

\section{Reduction to a left invertible system}

Consider a nonleft invertible system $\Sigma$. The main result of this section consists of a transformation of $\Sigma$, involving an algebraic state-feedback and an input static unit, so that the system thus obtained, say $\widehat{\Sigma}$, is left invertible, see Fig. 1. By performing a state-feedback with a friend $F$ of $\mathscr{V}_{\Sigma}^{*}$ assigning arbitrarily all the eigenvalues of $\left(A+B F \mid \mathscr{R}_{\Sigma}^{*}\right)$, and by eliminating all the input functions yielding motions on $\mathscr{R}_{\Sigma}^{*}$, we obtain a new system $\widehat{\Sigma}$ whose output-nulling reachable subspace $\mathscr{R}_{\widehat{\Sigma}}^{*}$ is the sole origin, and its invariant zeros are those of the original system, plus those assigned through $F$. It follows in particular that if $\Sigma$ is minimum phase, the modified system $\widehat{\Sigma}$ remains such with a suitable choice of $F$.

These results are presented and proved in the following theorem.

Theorem 1. Let $F$ be a friend of $\mathscr{V}_{\Sigma}^{*}$. Let $U_{s}$ be a basis matrix of the subspace $\mathscr{U}_{s}:=\left(B^{-1} \mathscr{V}_{\Sigma}^{*} \cap \operatorname{ker} D\right)^{\perp}$, whose dimension is denoted by $s$. Consider the quadruple $\widehat{\Sigma}:=(A+$ $\left.B F, B U_{s}, C+D F, D U_{s}\right)$. The following results hold:

1. $\mathscr{V}_{\Sigma}^{*}=\mathscr{V}_{\widehat{\Sigma}}^{*}$;

2. $\widehat{\Sigma}$ is left invertible;

3. $\mathscr{Z}(\widehat{\Sigma})=\mathscr{Z}(\Sigma) \uplus \sigma\left(A+B F \mid \mathscr{R}_{\Sigma}^{*}\right)$.

Proof. First, we denote by $\Sigma_{F}$ the quadruple $(A+B F, B, C+$ $D F, D)$. A straightforward computation shows that an outputnulling subspace of $\Sigma$ is also output-nulling for $\Sigma_{F}$ (and vice versa), i.e., $\mathscr{V}_{\Sigma}^{*}=\mathscr{V}_{\Sigma_{F}}^{*}$, [22, p. 169]. Now, we prove that $\mathscr{V}_{\Sigma_{F}}^{*}=$ $\mathscr{V}_{\widehat{\Sigma}}^{*}$. Notice that by definition of $\Sigma_{F}$ and $\widehat{\Sigma}$ the set of outputnulling subspaces of $\widehat{\Sigma}$ is contained in that of $\Sigma_{F}$ : in fact in $\widehat{\Sigma}$ the set of control functions is restricted with respect to those of $\Sigma_{F}$. Hence, we need to show that $\mathscr{V}_{\Sigma_{F}}^{*}$ is output nulling for $\widehat{\Sigma}$, i.e.,

$\left[\begin{array}{l}A+B F \\ C+D F\end{array}\right] \mathscr{V}_{\Sigma_{F}^{*}} \subseteq\left(\mathscr{V}_{\Sigma_{F}^{*}}^{*} \times 0_{p}\right)+\operatorname{im}\left[\begin{array}{c}B U_{s} \\ D U_{s}\end{array}\right]$.

To this end, apply the linear map $\left[\begin{array}{l}B \\ D\end{array}\right]$ to both sides of the trivial identity $\mathbb{R}^{m}=\left(B^{-1} \mathscr{V}_{\Sigma}^{*} \cap \operatorname{ker} D\right) \oplus \mathscr{U}_{s}$ to obtain

$\operatorname{im}\left[\begin{array}{l}B \\ D\end{array}\right] \subseteq\left(\left(\mathscr{V}_{\Sigma_{F}^{*}}^{*} \cap B \operatorname{ker} D\right) \times 0_{p}\right)+\operatorname{im}\left[\begin{array}{l}B \\ D\end{array}\right] U_{s}$.

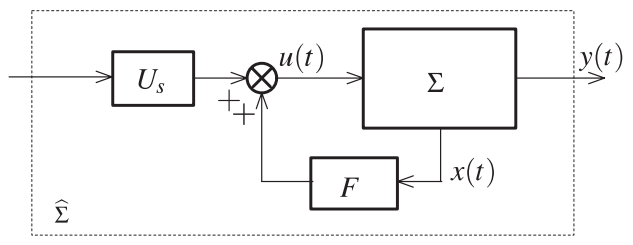

Fig. 1. Block diagram of a left inverter. 
Now, by adding the subspace $\mathscr{V}_{\Sigma_{F}^{*}}^{*} \times 0_{p}$ to both sides of the former we get

$\left(\mathscr{V}_{\Sigma_{F}}^{*} \times 0_{p}\right)+\operatorname{im}\left[\begin{array}{c}B \\ D\end{array}\right] \subseteq\left(\mathscr{V}_{\Sigma_{F}}^{*} \times 0_{p}\right)+\operatorname{im}\left[\begin{array}{c}B U_{s} \\ D U_{s}\end{array}\right]$,

which leads to (9). Now, by taking Lemma 3 into account, we show that $\widehat{\Sigma}$ is left invertible by proving that $\left(B U_{s}\right)^{-1} \mathscr{V}_{\widehat{\Sigma}}^{*} \cap$ $\operatorname{ker}\left(D U_{s}\right)=0_{s}$. Let $\omega \in\left(B U_{s}\right)^{-1} \mathscr{V}_{\widehat{\Sigma}}^{*} \cap \operatorname{ker}\left(D U_{s}\right)$. Thus, $B U_{s} \omega \in \mathscr{V}_{\widehat{\Sigma}}^{*}$ and $D U_{s} \omega=0_{p}$. Define $\tilde{\omega}:=U_{s} \omega$. The former yields $\tilde{\omega} \in B^{-1} \mathscr{V}_{\Sigma}^{*} \cap \operatorname{ker} D=\mathscr{U}_{s}^{\perp}$, since $\mathscr{V}_{\Sigma}^{*}=\mathscr{V}_{\widehat{\Sigma}}^{*}$. However, by definition $\tilde{\omega}$ lies in the range of $U_{s}$, hence it belongs to $\left(B^{-1} \mathscr{V}_{\Sigma}^{*} \cap \operatorname{ker} D\right)^{\perp}$. It follows that $\tilde{\omega}=0_{m}$. From the injectivity of $U_{s}$, we may conclude that $\omega=0_{s}$. As a result, $\widehat{\Sigma}$ is left invertible.

Finally, by definition of invariant zeros $\mathscr{Z}(\widehat{\Sigma})=\sigma(A+$ $\left.B F \mid \mathscr{V}_{\widehat{\Sigma}}^{*} / \mathscr{R}_{\widehat{\Sigma}}^{*}\right)$, which equals $\sigma\left(A+B F \mid \mathscr{V}_{\widehat{\Sigma}}^{*}\right)$ since $\widehat{\Sigma}$ is left invertible. However, since $\mathscr{V}_{\Sigma}^{*}=\mathscr{V}_{\widehat{\Sigma}}^{*}$, we get

$$
\begin{aligned}
\mathscr{Z}(\widehat{\Sigma}) & =\sigma\left(A+B F \mid \frac{\mathscr{V}_{\Sigma}^{*}}{\mathscr{R}_{\Sigma}^{*}}\right) \uplus \sigma\left(A+B F \mid \mathscr{R}_{\Sigma}^{*}\right) \\
& =\mathscr{Z}(\Sigma) \uplus \sigma\left(A+B F \mid \mathscr{R}_{\Sigma}^{*}\right) .
\end{aligned}
$$

So far, we have shown that the procedure described in Theorem 1 turns the given system into a left invertible system, whose invariant zeros are those of the original system, plus those given by $\sigma\left(A+B F \mid \mathscr{R}_{\Sigma}^{*}\right)$, which as already observed are all freely assignable with a suitable choice of the friend $F$ of $\mathscr{V}_{\Sigma}^{*}$. On the other hand, this procedure for the left inversion enjoys further important properties: it does not reduce the functional controllability properties of the original system, i.e., if $\Sigma$ is right invertible, so is the resulting system $\widehat{\Sigma}$ (and vice versa). Even more is true: the subspace $\mathscr{Q}_{\Sigma}^{*}=\mathscr{V}_{\Sigma}^{*}+\mathscr{S}_{\Sigma}^{*}$ is invariant under the transformation presented in Theorem 1. As already noted, this subspace is dual to $\mathscr{R}_{\Sigma}^{*}=\mathscr{V}_{\Sigma}^{*} \cap \mathscr{S}_{\Sigma}^{*}$. This result is presented and proved in the following proposition.

Proposition 1. Let $F$ be a friend of $\mathscr{V}_{\Sigma}^{*}$. Let $U_{s}$ be a basis matrix of the subspace $\mathscr{U}_{s}:=\left(B^{-1} \mathscr{V}_{\Sigma}^{*} \cap \operatorname{ker} D\right)^{\perp}$, whose dimension is denoted by $s$. Let $\widehat{\Sigma}:=\left(A+B F, B U_{s}, C+\right.$ $\left.D F, D U_{s}\right)$. Consider the sequences of subspaces $\left(\mathscr{S}_{\Sigma}^{i}\right)_{i \in \mathbb{N}}$ and $\left(\mathscr{S}_{\widehat{\Sigma}}^{i}\right)_{i \in \mathbb{N}}$. The following equality holds:

$\mathscr{V}_{\Sigma}^{*}+\mathscr{S}_{\Sigma}^{i}=\mathscr{V}_{\widehat{\Sigma}}^{*}+\mathscr{S}_{\widehat{\Sigma}}^{i}, \quad i \in \mathbb{N}$.

Hence, $\Sigma$ is right invertible if and only if $\widehat{\Sigma}$ is such. Moreover, $\widehat{\Sigma}$ and $\Sigma$ have the same zero structure at infinity and the same relative degree.

Proof. Denote by $\bar{\Sigma}$ the quadruple $\left(A, B U_{s}, C, D U_{s}\right)$. We first prove that $\mathscr{V}_{\Sigma}^{*}+\mathscr{S}_{\Sigma}^{i}=\mathscr{V}_{\bar{\Sigma}}^{*}+\mathscr{S}_{\bar{\Sigma}}^{i}$. To this aim, consider the two sequences of subspaces $\left(\mathscr{V}_{\Sigma}^{i}\right)_{i \in \mathbb{N}}$ and $\left(\mathscr{V}_{\bar{\Sigma}}^{i}\right)_{i \in \mathbb{N}}$ described in (4), which, respectively, converge to $\mathscr{V}_{\Sigma}^{*}$ and $\mathscr{V}_{\bar{\Sigma}}^{*}$ in at most $n-1$ steps. We first verify by induction that for any $i \in \mathbb{N}$ we have $\mathscr{V}_{\Sigma}^{i}=\mathscr{V}_{\bar{\Sigma}}^{i}$. This fact is clearly true when $i=0$. Let us now suppose that it holds for a given $i-1$, i.e., $\mathscr{V}_{\Sigma}^{i-1}=\mathscr{V}_{\bar{\Sigma}}^{i-1}$, and let us prove the same fact for $i$, i.e., $\mathscr{V}_{\Sigma}^{i}=\mathscr{V}_{\bar{\Sigma}}^{i}$. The following inclusion, that will be useful in the sequel, holds:

$\left[\begin{array}{c}B \\ D\end{array}\right] \mathscr{U}_{s}^{\perp} \subseteq\left(\mathscr{V}_{\Sigma}^{*} \cap B\right.$ ker $\left.D\right) \times 0_{p} \subseteq \mathscr{V}_{\Sigma}^{i-1} \times 0_{p}$

In fact, since sequence (4) is monotonically nonincreasing, $\mathscr{V}_{\Sigma}^{i-1} \supseteq \mathscr{V}_{\Sigma}^{*} \supseteq \mathscr{V}_{\Sigma}^{*} \cap B$ ker $D$. Now, from (4) we find

$$
\begin{aligned}
\mathscr{V}_{\Sigma}^{i} & =\left[\begin{array}{l}
A \\
C
\end{array}\right]^{-1}\left(\left(\mathscr{V}_{\Sigma}^{i-1} \times 0_{p}\right)+\left[\begin{array}{c}
B \\
D
\end{array}\right]\left(\mathscr{U}_{s}^{\perp} \oplus \mathscr{U}_{s}\right)\right) \\
& =\left[\begin{array}{l}
A \\
C
\end{array}\right]^{-1}\left(\left(\mathscr{V}_{\bar{\Sigma}}^{i-1} \times 0_{p}\right)+\left[\begin{array}{c}
B \\
D
\end{array}\right] \mathscr{U}_{s}\right),
\end{aligned}
$$

where the last equality is a consequence of (10). Hence, indeed $\mathscr{V}_{\Sigma}^{i}=\mathscr{V}_{\bar{\Sigma}}^{i}$. Now, we prove by induction that for any $i \in \mathbb{N}$, $\mathscr{S}_{\Sigma}^{i} \supseteq \mathscr{S}_{\bar{\Sigma}}^{i}$. Clearly, $\mathscr{S}_{\Sigma}^{0}=\mathscr{S}_{\bar{\Sigma}}^{0}$. Let us suppose that $\mathscr{S}_{\Sigma}^{i-1} \supseteq$ $\mathscr{S}_{\bar{\Sigma}}^{i-1}$ for a given $i$, and let us prove that $\mathscr{S}_{\Sigma}^{i} \supseteq \mathscr{S}_{\bar{\Sigma}}^{i}$. Let $\xi \in$ $\mathscr{S}_{\bar{\Sigma}}^{i}$. By (7), it follows that $\xi_{1} \in \mathscr{S}_{\bar{\Sigma}}^{i-1} \subseteq \mathscr{S}_{\Sigma}^{i-1}$ and $\xi_{2} \in \mathbb{R}^{s}$ exist such that $C \xi_{1}+D U_{s} \xi_{2}=0_{p}$ and $A \xi_{1}+B U_{s} \xi_{2}=\xi$. Define $\xi_{2}^{\prime}:=U_{s} \xi_{2}$. It follows that $\xi_{1} \in \mathscr{S}_{\Sigma}^{i-1}, C \xi_{1}+D \xi_{2}^{\prime}=0$, and $A \xi_{1}+B \xi_{2}^{\prime}=\xi$. The latter three relations clearly imply that $\xi \in \mathscr{S}_{\Sigma}^{i}$. Hence, we have shown that for any $i \in \mathbb{N}$ we have $\mathscr{S}_{\Sigma}^{i} \supseteq \mathscr{S}_{\bar{\Sigma}}^{i}$ and $\mathscr{V}_{\Sigma}^{i}=\mathscr{V}_{\bar{\Sigma}}^{i}$. A direct consequence of this result is that for any $i \in \mathbb{N}, \mathscr{V}_{\Sigma}^{i}+\mathscr{S}_{\Sigma}^{i} \supseteq \mathscr{V}_{\bar{\Sigma}}^{i}+\mathscr{S}_{\bar{\Sigma}}^{i}$. In order to show that the converse inclusion is true, i.e., that $\mathscr{V}_{\Sigma}^{i}+\mathscr{S}_{\Sigma}^{i} \subseteq$ $\mathscr{V}_{\bar{\Sigma}}^{i}+\mathscr{S}_{\bar{\Sigma}}^{i}$, we proceed by induction again. The inclusion holds for $i=0$. Let us suppose that $\mathscr{V}_{\Sigma}^{i-1}+\mathscr{S}_{\Sigma}^{i-1} \subseteq \mathscr{V}_{\bar{\Sigma}}^{i-1}+\mathscr{S}_{\bar{\Sigma}}^{i-1}$ for some defined $i$, and let us prove that $\mathscr{V}_{\Sigma}^{i}+\mathscr{S}_{\Sigma}^{i} \subseteq \mathscr{V}_{\bar{\Sigma}}^{i}+\mathscr{S}_{\bar{\Sigma}}^{i}$. Consider an arbitrary $\xi \in \mathscr{V}_{\Sigma}^{i}+\mathscr{S}_{\Sigma}^{i}$. It follows that $\xi_{1} \in \mathscr{S}_{\Sigma}^{i-1}$, $\xi_{2} \in \mathbb{R}^{m}$ and $\xi_{3} \in \mathscr{V}_{\Sigma}^{i}$ exist such that $C \xi_{1}+D \xi_{2}=0_{p}$ and $A \xi_{1}+B \xi_{2}+\xi_{3}=\xi$. Two vectors $\xi_{2}^{\prime} \in \mathscr{U}_{s}$ and $\xi_{2}^{\prime \prime} \in \mathscr{U}_{s}^{\perp}$ exist such that $\xi_{2}=\xi_{2}^{\prime}+\xi_{2}^{\prime \prime}$. Hence, $B \xi_{2}^{\prime \prime} \in \mathscr{V}_{\Sigma}^{*}$ and $D \xi_{2}^{\prime \prime}=0_{p}$. By linearity

$\xi=\left[\begin{array}{ll}A & B\end{array}\right]\left[\begin{array}{c}\xi_{1} \\ \xi_{2}^{\prime}+\xi_{2}^{\prime \prime}\end{array}\right]+\xi_{3}=\left[\begin{array}{ll}A & B\end{array}\right]\left[\begin{array}{l}\xi_{1} \\ \xi_{2}^{\prime}\end{array}\right]+\tilde{\xi}+\xi_{3}$,

holds, where $\tilde{\xi}:=\left[\begin{array}{ll}A & B\end{array}\right]\left[\begin{array}{l}0_{n} \\ \xi_{2}^{\prime \prime}\end{array}\right]$. On the other hand, as already observed, $D \xi_{2}^{\prime \prime}=0_{p}$. It follows that $C \xi_{1}+D \xi_{2}=C \xi_{1}+D \xi_{2}^{\prime}=$ $0_{p}$. Moreover, since $\xi_{2}^{\prime} \in \mathscr{U}_{s}$, a vector $\bar{\xi}_{2} \in \mathbb{R}^{s}$ exists such that $\xi_{2}^{\prime}=U_{s} \bar{\xi}_{2}$. Hence $C \xi_{1}+D U_{s} \bar{\xi}_{2}=0_{p}$ and, since $\mathscr{S}_{\Sigma}^{i-1}=\mathscr{S}_{\bar{\Sigma}}^{i-1}$, we find

$\left[\begin{array}{l}\xi_{1} \\ \xi_{2}\end{array}\right] \in\left(\mathscr{S}_{\bar{\Sigma}}^{i-1} \times \mathbb{R}^{S}\right) \cap \operatorname{ker}\left[\begin{array}{ll}C & D U_{s}\end{array}\right]$.

Finally, $\xi^{*}:=\tilde{\xi}+\xi_{3}=B \xi_{2}^{\prime \prime}+\xi_{3} \in \mathscr{V}_{\Sigma}^{*}+\mathscr{V}_{\Sigma}^{i}=\mathscr{V}_{\Sigma}^{i}$. We have shown that $\xi_{1} \in \mathscr{S}_{\bar{\Sigma}}^{i-1}, \bar{\xi}_{2} \in \mathbb{R}^{s}$ and $\xi_{3} \in \mathscr{V}_{\bar{\Sigma}}^{i}$ exist such that $A \xi_{1}+B U_{s} \bar{\xi}_{2}+\xi_{3}=\xi$ and $C \xi_{1}+D U_{s} \bar{\xi}_{2}=0_{p}$. This implies that $\xi \in \mathscr{V}_{\bar{\Sigma}}^{i}+\mathscr{S}_{\bar{\Sigma}}^{i}$. Hence, we have indeed $\mathscr{V}_{\Sigma}^{*}+\mathscr{S}_{\Sigma}^{*}=\mathscr{V}_{\bar{\Sigma}}^{*}+\mathscr{S}_{\bar{\Sigma}}^{*}$. Since $\mathscr{V}_{\Sigma}^{*}$ and $\mathscr{S}_{\Sigma}^{*}$ are invariant under state-feedback, [22, p. 169 , p. 183, it follows that $\mathscr{V}_{\Sigma}^{*}+\mathscr{S}_{\Sigma}^{*}=\mathscr{V}_{\Sigma_{F}^{*}}^{*}+\mathscr{S}_{\Sigma_{F}}^{*}$, where $\Sigma_{F}$ is defined as in the proof of Theorem 1. Hence, the procedure 
outlined above can be applied to $\Sigma_{F}$, yielding $\mathscr{V}_{\Sigma}^{*}+\mathscr{S}_{\Sigma}^{*}=$ $\mathscr{V}_{\Sigma_{F}}^{*}+\mathscr{S}_{\Sigma_{F}^{*}}^{*}=\mathscr{V}_{\widehat{\Sigma}}^{*}+\mathscr{S}_{\widehat{\Sigma}}^{*}$.

The last property that we want to investigate is the preservation of the stabilizability of the original system after reduction to a left invertible system.

Proposition 2. Let $\Sigma$ be a stabilizable system. Let $U_{s}$ be a basis matrix of the subspace $\mathscr{U}_{s}:=\left(B^{-1} \mathscr{V}_{\Sigma}^{*} \cap \operatorname{ker} D\right)^{\perp}$. Any friend $F$ of $\mathscr{V}_{\Sigma}^{*}$ for which $\sigma\left(A+B F \mid \mathscr{R}_{\Sigma}^{*}\right) \subset \mathbb{C}_{g}$ is such that the left invertible system $\widehat{\Sigma}:=\left(A+B F, B U_{s}, C+D F, D U_{s}\right)$ is stabilizable.

Proof. First, let us denote by $\mathscr{R}$ the reachable subspace from the origin, i.e., the smallest $A$-invariant subspace containing the range of $B$. We want to prove that the subspace $\mathscr{R}_{\Sigma}^{*}$ is externally stabilizable. Let $F$ be a friend of $\mathscr{R}_{\Sigma}^{*}$. From the trivial inclusion $\mathscr{R}_{\Sigma}^{*} \subseteq \mathscr{R}$ it follows that $\sigma\left(A+B F \mid \mathbb{R}^{n} /\left(\mathscr{R}+\mathscr{R}_{\Sigma}^{*}\right)\right)=\sigma(A+$ $\left.B F \mid \mathbb{R}^{n} / \mathscr{R}\right) \subset \mathbb{C}_{g}$, the last inclusion due to the stabilizability of $\Sigma$. It follows that $\mathscr{R}_{\Sigma}^{*}$ is externally stabilizable. We perform a preliminary change of coordinates in the state and input spaces through the nonsingular matrices $T=\left[\begin{array}{lll}T_{1} & T_{2} & T_{3}\end{array}\right] \in \mathbb{R}^{n \times n}$ and $U=\left[\begin{array}{ll}U_{1} & U_{2}\end{array}\right] \in \mathbb{R}^{m \times m}$, such that im $T_{1}=\mathscr{R}_{\Sigma}^{*}, \operatorname{im}\left[\begin{array}{ll}T_{1} & T_{2}\end{array}\right]=\mathscr{V}_{\Sigma}^{*}$, $\operatorname{im} U_{1}=B^{-1} \mathscr{V}_{\Sigma}^{*} \cap$ ker $D$ and im $U_{2}=\left(B^{-1} \mathscr{V}_{\Sigma}^{*} \cap \text { ker } D\right)^{\perp}$. If we take a friend $F$ of $\mathscr{V}_{\Sigma}^{*}$ assigning the eigenvalues $\sigma\left(A+B F \mid \mathscr{R}_{\Sigma}^{*}\right)$ in $\mathbb{C}_{g}$, the pair $(A+B F, B)$ can be written with respect to this basis as

$A+B F=\left[\begin{array}{ccc}A_{11}^{F} & A_{12}^{F} & A_{13}^{F} \\ 0 & A_{22}^{F} & A_{23}^{F} \\ 0 & 0 & A_{33}^{F}\end{array}\right], \quad B=\left[\begin{array}{cc}B_{11} & B_{12} \\ 0 & B_{22} \\ 0 & B_{32}\end{array}\right]$,

where the zeros in $A+B F$ are due to the $(A+B F)$-invariance of $\mathscr{V}_{\Sigma}^{*}$ and $\mathscr{R}_{\Sigma}^{*}$. The zeros in positions 21 and 31 in $B$ are due to the inclusion $\mathscr{V}_{\Sigma}^{*} \cap B$ ker $D \subseteq \mathscr{R}_{\Sigma}^{*}$. Since $\Sigma$ is stabilizable, such is the pair $(A+B F, B)$, so that the pair

$\left[\begin{array}{cc}A_{22}^{F} & A_{23}^{F} \\ 0 & A_{33}^{F}\end{array}\right], \quad\left[\begin{array}{l}B_{22} \\ B_{32}\end{array}\right]$,

is stabilizable, as well; let $\left[\begin{array}{ll}F_{1}^{\prime} & F_{2}^{\prime}\end{array}\right]$ be a feedback stabilizing the pair (11); it follows that the feedback $\tilde{F}:=\left[\begin{array}{lll}0 & F_{1}^{\prime} & F_{2}^{\prime}\end{array}\right]$ stabilizes the pair $\left(A+B F, B_{2}\right)$, where $B_{2}$ is the second blockcolumn of $B$, as $A_{11}^{F}$ is already stable. Now, since im $U_{2}=\operatorname{im} U_{s}$, we find that the system $\widehat{\Sigma}$ is stabilizable.

Remark 1. As is well-known, if the state of the given system is not accessible for the feedback, the block diagram of Fig. 1 can be replaced by the series connection of a system $\Sigma_{L}:=$ $\left(A+B F, B U_{s}, F, U_{s}\right)$ at the input of the given system $\Sigma$, in which $F$ is a friend of $\mathscr{V}_{\Sigma}^{*}$ and $U_{s}$ is a basis matrix of the subspace $\mathscr{U}_{s}=\left(B^{-1} \mathscr{V}_{\Sigma}^{*} \cap \operatorname{ker} D\right)^{\perp}$, see [4]. It is worth observing that the system $\Sigma_{L}$ introduces new invariant zeros in the overall system $\tilde{\Sigma}$, which are those that a suitable choice of the matrix $F$ would have added through the exploitation of a static state-feedback, plus the eigenvalues of $A$ which are, however, nonobservable (so that they are not transmission zeros of $\tilde{\Sigma}$ ). More precisely, consider the series connection of the given system $\Sigma$ with the quadruple $\Sigma_{L}=\left(A+B F, B U_{s}, F, U_{s}\right)$, in which $F$ is a friend of $\mathscr{V}_{\Sigma}^{*}$ and $U_{s}$ is a basis matrix of the subspace $\mathscr{U}_{s}=\left(B^{-1} \mathscr{V}_{\Sigma}^{*} \cap \operatorname{ker} D\right)^{\perp}$. The invariant zeros of the overall system $\tilde{\Sigma}$ are

$\mathscr{Z}(\tilde{\Sigma})=\left.\mathscr{Z}(\Sigma) \uplus \sigma(A) \uplus \sigma(A+B F)\right|_{\mathscr{R}_{\Sigma}^{*}}$.

The set of transmission zeros of $\tilde{\Sigma}$ equals the set of transmission zeros of the quadruple $\left(A+B F, B U_{s}, C+D F, D U_{s}\right)$.

\subsection{A numerical example}

Consider the example described by the following matrices:

$A=\left[\begin{array}{cccc}-3 & 5 & -7 & 0 \\ 0.5 & -1.5 & 0.5 & -7.5 \\ -5 & 0 & -3 & 0 \\ -0.5 & -5 & 0 & -7\end{array}\right], \quad B=\left[\begin{array}{ccc}1 & 0 & 0 \\ 0 & -1 & 0 \\ -2 & 0 & 0 \\ 0 & 1 & 2\end{array}\right]$,

$C=\left[\begin{array}{cccc}1 & 0 & 0 & 0 \\ 0 & -1 & 0 & 0\end{array}\right], \quad D=\left[\begin{array}{lll}1 & 0 & 0 \\ 2 & 0 & 0\end{array}\right]$.

System $\Sigma=(A, B, C, D)$ is nonleft invertible and right invertible. In fact

$\mathscr{V}_{\Sigma}^{*}=\operatorname{im}\left[\begin{array}{ccc}1 & 0 & 0 \\ -2 & 0 & 0 \\ 0 & 1 & 0 \\ 0 & 0 & 1\end{array}\right], \quad \mathscr{S}_{\Sigma}^{*}=\operatorname{im}\left[\begin{array}{ll}0 & 0 \\ 1 & 0 \\ 0 & 0 \\ 0 & 1\end{array}\right], \quad \mathscr{R}_{\Sigma}^{*}=\operatorname{im}\left[\begin{array}{l}0 \\ 0 \\ 0 \\ 1\end{array}\right]$.

In the present case the difference between the dimension of $\mathscr{V}_{\Sigma}^{*}$ and that of $\mathscr{R}_{\Sigma}^{*}$ is 2 , system $\Sigma$ has two invariant zeros, which are $\{-15.6589,-1.3411\}$, so that if $\Sigma$ is continuous-time, it is minimum phase. The first step consists of finding a friend $F \in$ $\mathbb{R}^{3 \times 4}$ of $\mathscr{V}_{\Sigma}^{*}$ assigning the eigenvalue of $(A+B F)$ restricted to $\mathscr{R}_{\Sigma}^{*}$. The matrix

$F=\left[\begin{array}{cccc}-3 & -1 & 0 & 0 \\ -3.5 & 10.5 & -13.5 & -7.5 \\ 1.2 & -2.4 & 5.4 & 6.25\end{array}\right]$

assigns the eigenvalue $\sigma\left(A+B F \mid \mathscr{R}_{\Sigma}^{*}\right)=\{-2\}$, and $\sigma(A+$ $\left.B F \mid \mathbb{R}^{n} /\left(\mathscr{V}_{\Sigma}^{*}+\mathscr{R}\right)\right)=\{-4\}$, where $\mathscr{R}$ is the reachable subspace from the origin. The matrix

$U_{s}=\left[\begin{array}{ll}1 & 0 \\ 0 & 1 \\ 0 & 0\end{array}\right]$

is a basis matrix of $\left(B^{-1} \mathscr{V}_{\Sigma}^{*} \cap \operatorname{ker} D\right)^{\perp}$. The new system $\Sigma_{1}$ described by the matrices $A_{1}=A+B F, B_{1}=B U_{s}, C_{1}=$ $C+D F, D_{1}=D U_{s}=0$ is left invertible, since $\mathscr{V}_{\Sigma_{1}}^{*}=\mathscr{V}_{\Sigma}^{*}$ but $\mathscr{S}_{\Sigma_{1}}^{*}=\operatorname{im}\left[\begin{array}{llll}0 & -1 & 0 & 1\end{array}\right]^{\top}$; moreover, the right invertibility is preserved. The set of its invariant zeros is exactly $\{-15.6589,-1.3411,-2\}$. Hence, $\Sigma_{1}$ is minimum phase.

\section{Conversion to a right invertible system and squaring down}

All the results presented so far can be easily dualized, so as to turn an arbitrary system $\Sigma$ into a right invertible system $\widehat{\Sigma}$, without affecting its functional observability properties. 


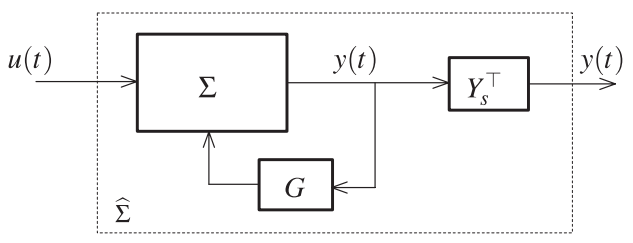

Fig. 2. Block diagram of a right inverter.

Clearly, this transformation involves an output injection via a matrix $G$ such that (6) hold, and assigning all the eigenvalues of $\left(A+G C \mid \mathbb{R}^{n} /\left(\mathscr{V}_{\Sigma}^{*}+\mathscr{S}_{\Sigma}^{*}\right)\right)$, and an output algebraic unit $Y_{s}^{\top}$, which is a basis matrix of the subspace $C \mathscr{S}_{\Sigma}^{*}+\operatorname{im} D$, see Fig. 2.

The following Corollary is the counterpart of Theorem 1, and Propositions 1 and 2 in the dual setting.

Corollary 1. Let $G$ be a friend of $\mathscr{S}_{\Sigma}^{*}$. Let $Y_{S}$ be a basis matrix of the subspace $C \mathscr{S}_{\Sigma}^{*}+\operatorname{im} D$. The quadruple $\widehat{\Sigma}:=(A+$ $\left.G C, B+G D, Y_{s}^{\top} C, Y_{s}^{\top} D\right)$ is such that

1. $\mathscr{S}_{\Sigma}^{*}=\mathscr{S}_{\widehat{\Sigma}}^{*}$;

2. $\widehat{\Sigma}$ is right invertible;

3. $\mathscr{Z}(\widehat{\Sigma})=\mathscr{Z}(\Sigma) \uplus \sigma\left(A+G C \mid \frac{\mathbb{R}^{n}}{\mathscr{V}_{\Sigma}^{*}+\mathscr{S}_{\Sigma}^{*}}\right)$;

4. $\mathscr{V}_{\Sigma}^{i} \cap \mathscr{S}_{\Sigma}^{*}=\mathscr{V}_{\widehat{\Sigma}}^{i} \cap \mathscr{S}_{\widehat{\Sigma}}^{*}, i \in \mathbb{N}$. Hence, in particular, $\widehat{\Sigma}$ is left invertible if and only if $\Sigma$ is such. Moreover, $\widehat{\Sigma}$ and $\Sigma$ have the same zero structure at infinity and the same relative degree.

Moreover, if $\Sigma$ is detectable, for any friend $G$ of $\mathscr{S}_{\Sigma}^{*}$ such that $\sigma\left(A+G C \mid \mathbb{R}^{n} / \mathscr{Q}_{\Sigma}^{*}\right) \subset \mathbb{C}_{g}$ the system $\widehat{\Sigma}$ is detectable, as well.

Hence, the set of invariant zeros of the resulting system $\widehat{\Sigma}$ is enlarged, so as to include the set of eigenvalues of $A+G C$ that are freely assignable through a suitable choice of $G$.

Furthermore, since the left reduction procedure preserves the right invertibility of the original system, while the right reduction procedure preserves the left invertibility, these two procedures can be applied together to an arbitrary system, possibly degenerate, so as to obtain a square and invertible system, described by the quadruple $\Sigma_{\mathrm{sq}}:=(A+B F+G C+G D F,(B+$ $\left.G D) U_{s}, Y_{s}^{\top}(C D F), Y_{s}^{\top} D U_{s}\right)$. Its set of invariant zeros includes the invariant zeros of the original system $\Sigma$. The matrices $F$ and $G$ can be chosen so as to place the additional invariant zeros introduced in the system $\Sigma_{\text {sq }}$ thus obtained at arbitrary locations of $\mathbb{C}_{g}$, as pointed out in Theorem 1 and Proposition 1.

Remark 2. In the case when the input of $\Sigma$ is not accessible for the output injection, the procedure outlined in Remark 1 can be dualized, so that a unit $\Sigma_{R}$, whose structure is dual to that of $\Sigma_{L}$, has to be connected at the output of $\Sigma$ in order to obtain an overall right invertible system.

\subsection{A numerical example}

The proposed approach turns out to be easily implementable as a software routine for the squaring down of a general LTI system. Consider the example described by the following matrices:

$$
\begin{aligned}
& A=\left[\begin{array}{cccc}
2 & 0 & 6 & 0 \\
0 & -5 & 0 & 12 \\
-2 & 0 & -8 & 0 \\
1 & -3 & 0 & 4
\end{array}\right], \quad B=\left[\begin{array}{cc}
1 & 0 \\
2 & 4 \\
0 & 0 \\
0 & -2
\end{array}\right], \\
& C=\left[\begin{array}{cccc}
1 & 0 & 2 & 0 \\
-3 & 0 & 0 & 0
\end{array}\right], \quad D=\left[\begin{array}{ll}
0 & 0 \\
0 & 0
\end{array}\right] .
\end{aligned}
$$

System $\Sigma=(A, B, C, D)$ is nonleft invertible and nonright invertible, since

$\mathscr{V}_{\Sigma}^{*}=\operatorname{im}\left[\begin{array}{ll}0 & 0 \\ 1 & 0 \\ 0 & 0 \\ 0 & 1\end{array}\right]$ and $\mathscr{S}_{\Sigma}^{*}=\operatorname{im}\left[\begin{array}{lll}1 & 0 & 0 \\ 0 & 1 & 0 \\ 0 & 0 & 0 \\ 0 & 0 & 1\end{array}\right]$.

In the present case $\mathscr{R}_{\Sigma}^{*}=\mathscr{V}_{\Sigma}^{*}$, so that $\Sigma$ has no invariant zeros. The first step consists of finding a friend $F \in \mathbb{R}^{2 \times 4}$ of $\mathscr{V}_{\Sigma}^{*}$ assigning the eigenvalues of $(A+B F)$ restricted to $\mathscr{R}_{\Sigma}^{*}$. The matrix

$F=\left[\begin{array}{cccc}0 & 0 & 7 & 0 \\ 0 & -0.5 & 0 & 0\end{array}\right]$

assigns the eigenvalues $\sigma\left(A+B F \mid \mathscr{R}_{\Sigma}^{*}\right)=\{-1,-2\}$, and $\sigma(A+$ $\left.B F \mid \mathbb{R}^{n} /\left(\mathscr{V}_{\Sigma}^{*}+\mathscr{R}\right)\right)=\{-3+i,-3-i\}$. The matrix $U_{s}=$ $(1 / \sqrt{10})\left[\begin{array}{ll}-1 & 3\end{array}\right]^{\top}$ is a basis matrix of $\left(B^{-1} \mathscr{V}_{\Sigma}^{*} \cap \operatorname{ker} D\right)^{\perp}$. The new system $\Sigma_{1}$ described by the matrices $A_{1}=A+B F$, $B_{1}=B U_{s}, C_{1}=C, D_{1}=D U_{s}=0$ is left invertible, but not right invertible, since $\mathscr{V}_{\Sigma_{1}}^{*}=\mathscr{V}_{\Sigma}^{*}$ but $\mathscr{S}_{\Sigma_{1}}^{*}=\left[\begin{array}{llll}-1 & -2 & 0 & 0\end{array}\right]$. The set of its invariant zeros is exactly $\{-1,-2\}$. Now, we derive a friend $G \in \mathbb{R}^{4 \times 2}$ of $\mathscr{S}_{\Sigma}^{*}$ such that $\sigma\left(A_{1}+G C_{1} \mid \mathbb{R}^{n} /\left(\mathscr{V}_{\Sigma}^{*}+\right.\right.$ $\left.\left.\mathscr{S}_{\Sigma}^{*}\right)\right)=\{-4,-5\}$. In this case there are no internal eigenvalues $\sigma\left(A_{1}+G C_{1} \mid \mathscr{S}_{\Sigma}^{*} /\left(\mathscr{S}_{\Sigma}^{*} \cap \mathscr{Q}_{\Sigma}^{*}\right)\right)$ to assign:

$G=\left[\begin{array}{cc}-2.0148 & 1.6617 \\ 1.0574 & -0.9809 \\ 2 & 0 \\ 0.0652 & -0.9783\end{array}\right]$

The matrix $Y_{S}=(1 / \sqrt{10})\left[\begin{array}{ll}-1 & 3\end{array}\right]^{\top}$ is a basis matrix of $C_{1} \mathscr{S}_{\Sigma_{1}}^{*}+$ im $D$. The system $\Sigma_{2}$ described by the matrices $A_{2}:=A_{1}+$ $G C_{1}, B_{2}:=B_{1}+G D_{1}, C_{2}:=Y_{s}^{\top} C_{1}, D_{2}:=Y_{s}^{\top} D_{1}$ :

$$
\begin{aligned}
& A_{2}=\left[\begin{array}{cccc}
-5 & 0 & 8.9704 & 0 \\
4 & -7 & 16.1148 & 15 \\
0 & 0 & -4 & 0 \\
4 & -2 & 0.1304 & 4
\end{array}\right], \quad B_{2}=\left[\begin{array}{c}
-1 \\
-2 \\
0 \\
0
\end{array}\right], \\
& C_{2}=\left[\begin{array}{llll}
-3.1623 & 0 & -0.6325 & 0
\end{array}\right], \quad D_{2}=0,
\end{aligned}
$$

is therefore left and right invertible, since $\mathscr{R}_{\Sigma_{2}}^{*}=0_{n}$ and $\mathscr{V}_{\Sigma_{2}}^{*}+$ $\mathscr{S}_{\Sigma_{2}}^{*}=\mathbb{R}^{n}$, and is minimum phase, since its invariant zeros are $\{-1,-2,-4\}$.

\section{The LQ optimal control problem}

As an example of application of the results presented so far, we present a parametrization of the solutions of the LQ problem for nonleft invertible systems. Consider for example the discrete-time nonleft invertible system $\Sigma$ with assigned initial 
condition described by

$x(k+1)=A x(k)+B u(k), \quad x(0)=x_{0} \in \mathbb{R}^{n}$,

$y(k)=C x(k)+D u(k)$.

The problem is that of finding a stabilizing state-feedback $u(k)=-K_{+} x(k)$ minimizing the performance index

$J\left(x_{0}, u\right)=\sum_{k=0}^{\infty} y^{\top}(k) y(k)$,

under the constraint (12). In this case, the optimal solution is not unique. In fact, since in this case $\mathscr{R}_{\Sigma}^{*}$ differs from zero, dinstinct control functions exist yielding state trajectories corresponding to identically zero output functions. In other words, given an optimal control law $u^{o}(k)$, the set of optimal control functions is parametrized modulo the controls driving the state of $\Sigma$ on $\mathscr{R}_{\Sigma}^{*}$. Let $Q:=C^{\top} C, S:=C^{\top} D$ and $R:=D^{\top} D$. Since $\Sigma$ is not left invertible, the extended symplectic pencil associated with the LQ problem is singular, so that the discrete-time algebraic Riccati equation (DARE)

$P=A^{\top} P A-\left(A^{\top} P B+S\right)\left(R+B^{\top} P B\right)^{-1}\left(B^{\top} P A+S^{\top}\right)+Q$,

does not admit a stabilizing solution, [10]. Now, consider the system $\widehat{\Sigma}=(\widehat{A}, \widehat{B}, \widehat{C}, \widehat{D}):=\left(A+B F, B U_{s}, C+D F, D U_{s}\right)$, where $F$ is a friend of $\mathscr{V}_{\Sigma}^{*}$ and $U_{s}$ is a basis matrix of the subspace $\left(B^{-1} \mathscr{V}_{\Sigma}^{*} \cap \operatorname{ker} D\right)^{\perp}$. System $\widehat{\Sigma}$ is now left invertible by virtue of Theorem 1. Hence, the infinite-horizon LQ problem can be solved with respect to the auxiliary system $\widehat{\Sigma}$ : in particular, the stabilizing solution $\widehat{P}_{+}$of the DARE (13) referred to $\widehat{\Sigma}$ yields the optimal gain $\widehat{K}_{+}:=\left(\widehat{R}+\widehat{B}^{\top} \widehat{P}_{+} \widehat{B}\right)^{-1}\left(\widehat{B}^{\top} \widehat{P}_{+} \widehat{A}+\right.$ $\widehat{S}^{\top}$ ), where $\widehat{Q}:=\widehat{C}^{\top} \widehat{C}, \widehat{S}:=\widehat{C}^{\top} \widehat{D}$ and $\widehat{R}:=\widehat{D}^{\top} \widehat{D}$. Then, the matrix $K_{+}=U_{s} \widehat{K}_{+}-F$ is a gain leading to an optimal control function. Different choices of the matrix $F$ yield different solutions of the LQ problem, corresponding to the possibility of assigning arbitrarily the eigenvalues of $(A+B F)$ on $\mathscr{R}_{\Sigma}^{*}$, so that the state evolutions are different, but they all correspond to the same output function, and the value of the performance index does not change and is equal to its optimal value $J^{*}$. Hence, all optimal solutions of the LQ problem herein considered can be parametrized in terms of all possible friends $F$ of $\mathscr{V}_{\Sigma}^{*}$ such that $\sigma\left(A+B F \mid \mathscr{R}_{\Sigma}^{*}\right) \subset \mathbb{C}_{g}$, without the need of resorting to the stabilizing solution of the generalized discrete algebraic Riccati equation. It is worth observing that the same technique can be employed for a parametrization of the solutions of continuous-time singular LQ problems, in which the assumption of left invertibility is standard, [9].

\subsection{An illustrative example}

Consider a discrete-time system $\Sigma$ with assigned initial condition described by the matrices

$$
\begin{aligned}
& A=\left[\begin{array}{ll}
1 & 1 \\
0 & 1
\end{array}\right], \quad B=\left[\begin{array}{ll}
2 & 0 \\
1 & 1
\end{array}\right], \quad x(0)=\left[\begin{array}{c}
1 \\
-1
\end{array}\right], \\
& C=\left[\begin{array}{ll}
0 & 1
\end{array}\right], \quad D=\left[\begin{array}{ll}
0 & 0
\end{array}\right] .
\end{aligned}
$$

This system is nonleft invertible, since $\mathscr{R}_{\Sigma}^{*}=\operatorname{im}\left[\begin{array}{ll}1 & 0\end{array}\right]^{\top}$, but it is right invertible since $\mathscr{S}_{\Sigma}^{*}=\mathbb{R}^{n}$. Let $Q:=C^{\top} C, S:=C^{\top} D$ and $R:=D^{\top} D$. Since $\Sigma$ is not left invertible, the DARE does not admit a stabilizing solution. Consider the matrices

$F=\left[\begin{array}{cc}-\frac{3}{4} & -\frac{1}{4} \\ \frac{3}{4} & -\frac{1}{4}\end{array}\right], \quad U_{s}=\left[\begin{array}{l}1 \\ 1\end{array}\right]$,

where $F$ is a friend of $\mathscr{V}_{\Sigma}^{*}$, assigning $\sigma\left(A+B F \mid \mathscr{R}_{\Sigma}^{*}\right)=\left\{-\frac{1}{2}\right\}$ and $\sigma\left(A+B F \mid \mathbb{R}^{n} /\left(\mathscr{V}_{\Sigma}^{*}+\mathscr{R}\right)\right)=\left\{\frac{1}{2}\right\}$, where $\mathscr{R}$ is the reachable subspace from the origin, while $U_{s}$ is a basis matrix for $\left(B^{-1} \mathscr{V}_{\Sigma}^{*}\right)^{\perp}$. The new system described by

$\widehat{A}=A+B F=\left[\begin{array}{cc}-\frac{1}{2} & \frac{1}{2} \\ 0 & \frac{1}{2}\end{array}\right], \quad \widehat{B}=B U_{s}=\left[\begin{array}{l}2 \\ 2\end{array}\right]$,

$\widehat{C}=C+D F=\left[\begin{array}{ll}0 & 1\end{array}\right], \quad \widehat{D}=D U_{s}=[0]$

is now left invertible. The DARE written with respect to $\widehat{A}$, $\widehat{B}, \widehat{Q}:=\widehat{C}^{\top} \widehat{C}, \widehat{S}:=\widehat{C}^{\top} \widehat{D}$ and $\widehat{R}:=\widehat{D}^{\top} \widehat{D}$, admits a stabilizing solution and an optimal infinite-horizon gain, which are, respectively,

$\widehat{P}_{+}=\left[\begin{array}{ll}0 & 0 \\ 0 & 1\end{array}\right], \quad \widehat{K}_{+}=\left[\begin{array}{ll}0 & \frac{1}{4}\end{array}\right]$.

The optimal gain referred to the original system is

$K_{+}=U_{s} \widehat{K}_{+}-F=\left[\begin{array}{cc}\frac{3}{4} & \frac{1}{2} \\ -\frac{3}{4} & \frac{1}{2}\end{array}\right]$.

The overall optimal cost is $J^{*}=x_{0}^{\top} \widehat{P}_{+} x_{0}=1$. As we could expect, if we change $F$ in order to choose for example $-\frac{1}{5}$ as internal and $-\frac{1}{2}$ as external assignable eigenvalues of $\mathscr{V}_{\Sigma}^{*}$, respectively, we find the same solution $\widehat{P}_{+}$of (13) applied to $\widehat{\Sigma}$, hence leading to the same cost, with a different solution $\widehat{K}_{+}$. Hence, the optimal gain referred to the original system $K_{+}^{\prime}$ is, in this case,

$K_{+}^{\prime}=U_{s} \widehat{K}_{+}-F=\left[\begin{array}{cc}\frac{3}{5} & 1 \\ -\frac{3}{5} & 1\end{array}\right]$.

\section{Concluding remarks}

A geometric setting has been established for the solution of the left and right inversion of nonstrictly proper LTI systems, leading to a simple and computationally attractive procedure for the squaring down problem. It has been proved that the squared-down system is minimum phase if so is the original system, and the invariant zeros induced by the pre- and post-compensators are all freely assignable in the complex plane. The approach developed for the left and right reduction presented in Sections 4 and 5 has been implemented as an algorithm in the MATLAB ${ }^{\circledR}$ routines leftinv.m and rightinv.m, available ${ }^{1}$ at http://www. dii.unisi.it/prattichizzo/research/geometric/leftrightinv.html.

\footnotetext{
1 The MATLAB ${ }^{\circledR}$ routines leftinv.m and rightinv.m require the geometric approach toolbox $g a$, which is freely downloadable at www3.deis.unibo.it/Staff/FullProf/GiovanniMarro/geometric.htm.
} 
These new techniques are not merely oriented to the solution of the aforementioned problems, but highlight important geometric properties of LTI systems that can be exploited for the solution of different control problems; as an example we have considered the infinite-horizon LQ problem for discrete-time nonleft invertible systems: the left inversion procedure yields a parametrization of all the optimal solutions in terms of motions on a subspace corresponding to modes that are not penalized in the performance index.

Current limitation of this work is that the proposed squaring down techniques require the state to be available. Work is in progress to investigate the behaviour of the proposed techniques for problems where the state is not available and the system is detectable.

\section{Ackowledgements}

The authors wish to thank Prof. Giovanni Marro for his precious and helpful comments.

\section{References}

[1] H. Aling, J.M. Schumacher, A nine-fold canonical decomposition for linear systems, Internat. J. Control 39 (4) (1984) 779-805.

[2] J.D. Aplevich, On the squaring problem in feedback design, in: Proceedings of the Joint Automatic Control Conference, vol. 43.6, Charlottesville, 1981, pp. WP-4P.

[3] G. Basile, G. Marro, A new characterization of some structural properties of linear systems: unknown-input observability, invertibility and functional controllability, Internat. J. Control 17 (5) (1973) 931-943.

[4] G. Basile, G. Marro, Controlled and Conditioned Invariants in Linear System Theory, Prentice-Hall, Englewood Cliffs, New Jersey, 1992.

[5] D. Chu, Y.S. Hung, A numerically reliable solution for the squaring-down problem in system design, Appl. Numer. Math. 51 (2004) 221-241.

[6] D.J. Clements, B.D.O. Anderson, Singular optimal control: the linearquadratic problem, Springer, Berlin, New York, 1975.
[7] C. Commault, J.M. Dion, Structure at infinity of linear multivariable systems: a geometric approach, IEEE Trans. Automat. Control AC-27 (3) (1982) 693-696.

[8] E.J. Davison, Some properties of minimum phase systems and 'squareddown' systems, IEEE Trans. Automat. Control AC-28 (1983).

[9] M.L.J. Hautus, L.M. Silverman, System structure and singular control, Linear Algebra Appl. 50 (1983) 369-402.

[10] V. Ionescu, C. Oara, M. Weiss, Generalized Riccati theory and robust control, a Popov function approach, Wiley, New York, 1999.

[11] D.H. Jacobson, Totally singular quadratic minimization problems, IEEE Trans. Automat. Control AC-16 (1971) 651-658.

[12] V.X. Le, M.G. Safonov, Rational matrix GCD's and the design of squaring-down compensator-a state space theory, IEEE Trans. Automat. Control AC-37 (3) (1992) 384-392.

[13] A.G.J. MacFarlane, N. Karcanias, Poles and zeros of linear multivariable systems: a survey of the algebraic, geometric and complex variable theory, Internat. J. Control (1976) 33-74.

[14] G. Marro, D. Prattichizzo, E. Zattoni, A geometric insight into the discrete time cheap and singular LQR problems, IEEE Trans. Automat. Control 47 (1) (2002) 102-107.

[15] A.S. Morse, Structural invariants of linear multivariable systems, SIAM J. Control 11 (3) (1973) 446-465.

[16] A. Saberi, P. Sannuti, Squaring down of non-strictly proper systems, Internat. J. Control 51 (3) (1990) 621-629.

[17] P. Sannuti, A. Saberi, Special coordinate basis for multivariable linear systems-finite and infinite zero structure, squaring down and decoupling, Internat. J. Control 45 (5) (1987) 1655-1704.

[18] P. Sannuti, A. Saberi, Squaring down by static and dynamic compensators, IEEE Trans. Automat. Control AC-33 (4) (1988) 358-365.

[19] A.A. Stoorvogel, The singular $H_{2}$ control problem, Automatica 28 (3) (1992) 627-631.

[20] A.A. Stoorvogel, J.H.A. Ludlage, Squaring down and the problems of almost zeros for continuous-time systems, Systems \& Control Lett. 23 (5) (1994) 381-388.

[21] G. Tadmor, L. Mirkin, $H_{\infty}$ control and estimation with preview-part II: fixed-size ARE solutions in discrete time, IEEE Trans. Automat. Control AC-50 (1) (2005) 29-40.

[22] H.L. Trentelman, A.A. Stoorvogel, M. Hautus, Control theory for linear systems, Comm. Control Eng., Springer, Great Britain, 2001.

[23] W.M. Wonham, Linear Multivariable Control: A Geometric Approach, third ed., Springer, New York, 1985. 